\title{
评 述＼cjkstart强场和原子分子物理专题
}

\section{原子发射高次谐波的优化控制和动力学分析}

\author{
王国利 $1^{*}$ ，李鹏程 $1^{*}$ ，李小勇 ${ }^{2}$ ，赵松峰 ${ }^{1}$ ，周效信 $1^{*}$ \\ 1. 西北师范大学物理与电子工程学院, 兰州 730070 ; \\ 2. 西北民族大学实验中心, 兰州 730030 \\ * 联系人, 王国利, E-mail: wanggl@nwnu.edu.cn; 李鹏程, E-mail: lipc@nwnu.edu.cn; 周效信, E-mail: zhouxx@nwnu.edu.cn
}

摘要原子分子在强激光场中发射的高次谐波是一种新型的便捷型光源, 目前已在众多研究领域展现出 了非常诱人的应用前景. 本文综述了针对高次谐波转换效率低而对其进行优化控制的常用方法和结果, 结合 波形整形技术和遗传算法等演化算法, 人们可以对高次谐波进行各种目的性的控制, 特别是利用近年发展成 熟的波形合成技术, 可将谐波场的强度提高 1 个数量级以上, 或者在不降低强度的基础上将谐波截止位置扩 展 2 倍以上. 最后介绍了一种全新的用于高次谐波分析的时频变换方法——同步压缩技术 (Synchrosqueezing Transform, SST), 该变换技术为理解和探索电离阈值附近及以下高次谐波发射的动力学过程提供了强有力的 工具.

关键词高次谐波, 优化控制, 脉冲整形,波形合成,动力学分析

PACS: 42.65.Ky, 42.65.Re, 32.80.Qk

\section{1 引言}

高次谐波产生 (High-order harmonic generation, HHG) 是一种重要的频率转换过程: 一束高强度 $\left(>10^{14} \mathrm{~W} / \mathrm{cm}^{2}\right)$ 的飞秒激光与原子、分子相互作用 而释放出频率为激光频率高阶倍的高能光子. 其发 射机理可以用 “三步模型” 来进行描述 ${ }^{[1]}$. 自从 20 世纪 80 年代末 ${ }^{[2,3]}$ 实验中成功观察到高次谐波后, 关于 HHG 的研究迅速成为强场物理的一个重要研 究领域 ${ }^{[4]}$. 由于这些谐波具有频率可调谐、高相干
性的优点, 它们在诸多研究领域都展现出了诱人的 应用前景. 如利用高次谐波获得迄今为止时间脉宽 最短的激光—阿秒脉冲 [5]、进行 X 射线衍射成 像 [6-8]、用于时间分辨的泵浦 - 探测光谱学 [9-11]、 研究合金材料的磁结构和磁化动力学 [11-14]、提取

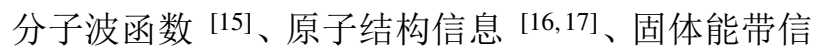
息 ${ }^{[18,19]}$ 以及激光脉冲的时间信息 (包络和啁啾) ${ }^{[20]}$ 等. 最近, $\mathrm{X}$ 射线波段圆偏振高次谐波的成功产 生 [21-24] 为研究材料的磁圆二向色性提供了重要的 研究工具.

引用格式: 王国利, 李鹏程, 李小勇, 等. 原子发射高次谐波的优化控制和动力学分析. 中国科学: 物理学 力学 天文学, 2017, 47: 033002 Wang G L, Li P C, Li X Y, et al. The optimal control and dynamical analysis of high-order harmonic generation from atom (in Chinese). Sci Sin-Phys Mech Astron, 2017, 47: 033002, doi: 10.1360/SSPMA2016-00251 
目前影响高次谐波广泛应用的一个主要瓶颈 是它的光通量很低, 对于常用的 Ti:sapphire 激光 $(800 \mathrm{~nm})$ 而言谐波的转换效率通常小于 $10^{-5}$ [25-27]. 若使用波长更长的红外激光可以有效提高光子的最 大能量 一 截止位置, 但是转换效率急剧下降 (单原 子 $\sim \lambda^{-5}$ ). 从实际应用的角度来看, 高次谐波的产生 需要解决以下几个问题 ${ }^{[28]}$ : 提高转换效率、扩展截 止位置、选择性增强和抑制某阶或某一范围的谐波 (见图 1).

实验中观察到的高次谐波通常都是在稠密的气 体中产生, 它不仅与单个原子在激光场中的响应 (诱 导偶极矩) 有关 ${ }^{[29]}$, 还与激光场和每个原子产生的 谐波场在介质中的传播特性有关, 需考虑介质对它 们的吸收、色散以及相位匹配等效应 ${ }^{[30,31]}$. 因此, 从高次谐波的产生过程来看, 可以通过改变激光脉 冲的波形和实验条件两种方式来对其进行优化控制. 本文将对一些主要的方法和结果作一综述. 这些结 果通常借助于某种演化算法来实现, 最常用的是遗 传算法 ${ }^{[32]}$ (Genetic Algorithm, GA) 或者优化控制理

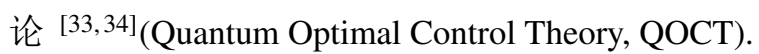

另外, 考虑到时频分析是理解高次谐波发射动 力学过程的一种重要手段, 我们还将介绍近来成功 用于分析电离阈值附近 HHG 动力学过程的同步压缩

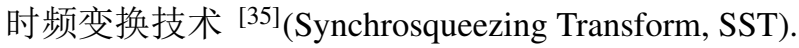
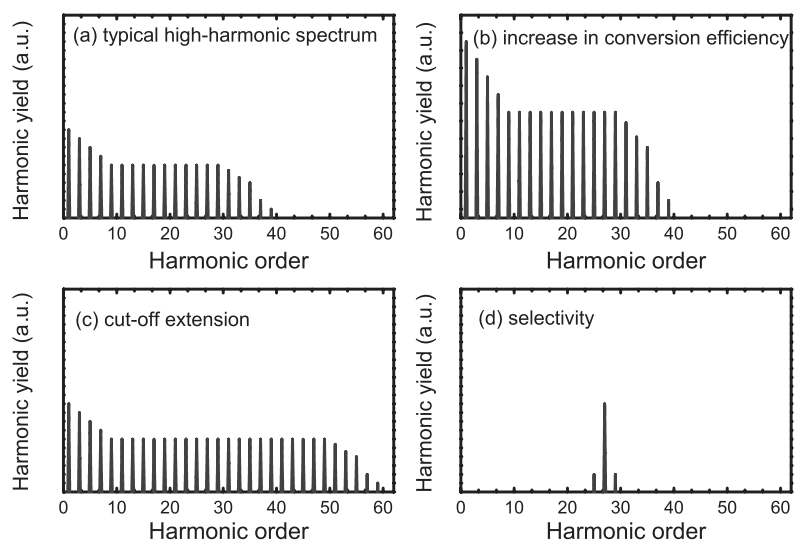

图 1 高次谐波谱的特征 (a) 及其面临的挑战 ${ }^{[28]}$ : (b) 提高转 换效率; (c) 扩展截止位置; (d) 选择性增强和抑制

Figure 1 The characteristics of the high-harmonic (a) and the challenges for further experiments [28]: (b) extension of the cutoff energy; (c) increase in the efficiency of the high-harmonic conversion process; and (d) the selection of single harmonics or range of harmonics.

\section{2 高次谐波发射的优化控制}

\section{1 波形整形技术}

通过脉冲整形技术 [36,37] 获得任意形状的飞秒 激光进而控制高次谐波的产生是较早用于优化 HHG 的方法之一,这种优化可以分为时域整形和空间整形 来实现.

\subsection{1 时域整形}

一束时域中的脉冲可表示为

$E(t)=E_{\text {env }}(t) \mathrm{e}^{\mathrm{i} \omega_{0} t}$,

其中包络 $E_{\mathrm{env}}$ 决定了脉冲的振幅和脉宽. 根据傅里 叶变换, 可将其写为

$E(t)=\frac{1}{2 \pi} \sum_{j=1}^{n} E\left(\omega_{j}\right) \mathrm{e}^{-\mathrm{i} \varphi_{j}} \mathrm{e}^{\mathrm{i} \omega_{j} t} \Delta \omega_{j}$.

因此, 首先利用色散系统将激光脉冲变换到频 域, 通过改变不同频率成份的相位 $\varphi_{j}$ (可通过一个可 变形反射镜改变光程来实现), 就可改变脉冲在时域 中的振幅和相位. 因为辐射光子的相位与其对应电 子电离时刻激光场的强度和相位有关, 所以利用这 种时域整形技术可以使某阶谐波对应的所有轨道具 有相同的位相而实现干涉相长——原子内相位匹 配, 从而实现对特定阶次谐波的选择性增强 [38-41].

虽然理论结果表明利用脉冲整形技术可以实现 单原子谐波的选择性增强 ${ }^{[39,40]}$, 但是实验研究表明 利用气体喷嘴并不能实现这种选择性控制, 而利用 中空光纤不仅能实现对特定谐波的增强, 还能实现

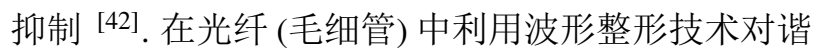
波进行选择性控制已在文献 $[28,43]$ 中给出了完整 的综述. 需要补充说明的是, 最近的实验 ${ }^{[44,45]}$ 和理 论 ${ }^{[46]}$ 研究结果表明, 通过优化双色、三色激光场的 啁啾和相位也可以在气体喷嘴或者气室 (Cell) 中实 现对特定阶次谐波的选择性增强.

另外一种剪裁激光波形的方式是采用可编程的 声光色散滤波器 ${ }^{[47,48]}$. 利用优化的激光脉冲与气室 作用, 不仅可以提高谐波的效率, 还可以对特定谐波 的波长进行调谐 ${ }^{[47]}$. 通过在不同实验条件 (压强、聚 焦位置、聚光圆孔的大小) 下优化谐波通量, 表明这 
种时域整形的最优波形并不直接依赖于宏观相位匹 配 [48].

\subsection{2 空间整形}

利用可变形反射镜 $[49,50]$ 或者液晶空间光调制 器 [51,52] 可以控制激光脉冲的位相而校正波前形状. 通过操控聚焦处的强度和相位可以优化激光脉冲引 入的几何相位和原子偶极矩相位 $(\propto I)$, 进而优化宏 观相位匹配条件 ${ }^{[50]}$, 或者增大作用区域激光束的面 积 ${ }^{[51]}$ 、强度 ${ }^{[49]}$ 从而提高电离场来增强气体喷嘴 产生的整个平台区谐波的强度, 而且可以扩展截止 位置.

若利用剪裁的激光脉冲与充满气体的光纤作用, 则可以激发光纤的不同工作模式, 从而可以将整个 谐波场强度提高 1 个数量级 ${ }^{[52]}$.

\section{2 优化激光啁啾}

根据高次谐波的产生机理, 其产生的 XUV 光谱 具有很大的啁啾 ${ }^{[53-55]}$. 对某阶谐波来说, 对应于长 短轨道的相位为

$\Phi_{i}(r, z, t)=-\alpha_{i} I(r, z, t)$,

其中, $i=1,2$, 分别代表长短轨道. 时域激光强度 $I(t)$ 的 变化会产生啁啾 $\Delta \omega_{i}(t)=-\partial \Phi_{i}(t) / \partial t$. 该啁啾会加宽 谐波谱从而降低峰值强度. 为了控制谐波产生, 可用 激光啁啾来补偿该动力学诱导的啁啾. 实验表明, 采 用合适啁啾的激光脉冲, 谐波的峰值强度会得到明 显提高 $\left(\sim 5\right.$ 倍) ${ }^{[56,57]}$.

另外, 啁啾可以有效改变激光脉冲的对称性, 从 而非常有利于单个阿秒脉冲的产生 ${ }^{[58,59]}$. 理论研 究表明, 通过优化啁啾以及载波包络相位可以有效 改善阿秒脉冲的质量 — 更高的强度和更窄的脉 宽 ${ }^{[60-62]}$.

\section{3 优化实验条件 一一 宏观相位匹配}

宏观高次谐波的产生受介质吸收和相位匹配条 件的限制. 而这些效应与气体靶长度、压强、位置、 脉冲强度等因素都有关系, 如压强不仅能改变谐波 谱的结构, 还会影响谐波场的强度. 对每一阶谐波而
言, 为了得到最强的谐波场, 都存在一个最优的压强, 而且该压强值依赖于激光强度和气体靶长度 ${ }^{[63,64]}$. 理论模拟表明, 同时优化多个实验参数 (压强、气 体靶的长度和位置) 可以有效提高谐波强度、压缩

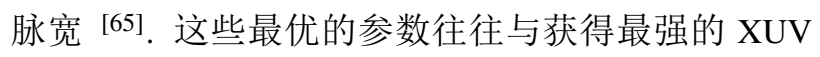
光通量 (Flux) 和最强亮度 (Brilliance) 的条件是一致 的 [66]. 最近的实验结果证实通过优化气体靶位置可 使截止位置处的谐波强度提高 1 个数量级 ${ }^{[67]}$.

要指出的是, 虽然利用各种方式可以有效提高 气体喷嘴产生谐波的效率或截止位置 (见下节), 由 于充气波导管更易满足完全相位匹配条件 ${ }^{[68]}$, 所以 实验室最可能产生高强度、能量达 $\mathrm{keV}$ 的谐波的几 何构型应该是波导管.

\section{4 波形合成技术}

与前面提及的波形整形技术不同的是, 波形合 成技术指的是通过精确控制几束激光脉冲的参数 (相对波长、振幅、相位) 来产生组合激光场 [69-74]. 利用这种技术得到的激光场波形更加多样化 ${ }^{[69]}$. 接 下来重点介绍这种技术在优化 HHG 方面 (图 1(b) 和 (c)) 的应用.

\subsection{1 提高 HHG 的转换效率}

许多实验研究工作 ${ }^{[75-84]}$ 都表明利用多色激光 场可以有效提高谐波场的强度, 下面将说明在激光 总能量和谐波截止位置给定的前提下, 利用优化的 双色场来提高谐波转换效率的理论模拟结果.

一般通过波形合成来提高谐波场的基本思想是: 增加电子电离时刻电场的强度来释放更多的电子、 增强谐波形成过程中电子短轨道的贡献 ${ }^{[85]}$. 从这种 思想出发, Jin 等人 ${ }^{[85]}$ 优化了最优双色、三色场的 波形. 与单色场相比, 在电离几率 $<5 \%$ 、激光总能量 仅增加 $10 \%$ 、具有相同截止位置的前提下, 优化场产 生单原子谐波场的强度可提高约 2 个数量级, 宏观 谐波场增强的程度甚至更加显著. 通过直接优化谐 波场, 如图 2 所示, 我们的结果 ${ }^{[86]}$ 也证实了 Jin 等人 的结果, 而且分析还表明 (图 2(b)), 在单色场和双色 场中电子运动导致的波包扩散也基本相同. 我们的 优化还表明, 最优波形与激光波长和强度几乎没有 

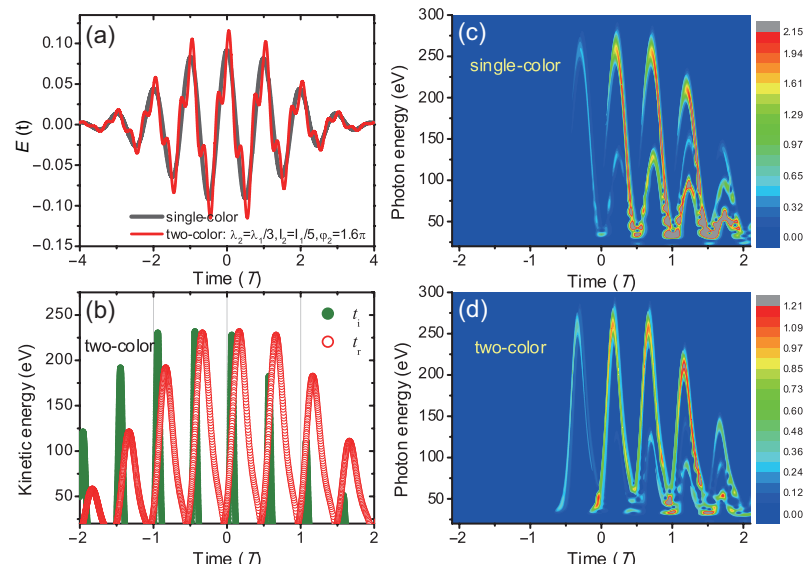

图 2 (网络版彩图) 通过波形合成来增强谐波场的基本思

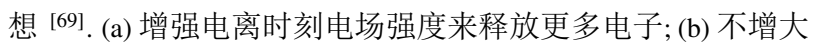
电子波包扩散; (c), (d) 增强电子短轨道贡献

Figure 2 (Color online) The strategies for modifying the waveform to enhance HHG yield [69]. (a) Increase the laser's electric fields to release more electrons; (b) the spreading of the electron wavepacket is not increased; (c), (d) enhance the emissions from short-trajectory electrons.

依赖性. 基频场波长 $\lambda_{1}=(800-3000) \mathrm{nm}$, 在总 强度固定的条件下, 最优双色场波形的参数为 $\lambda_{2}=$ $\lambda_{1} / 3-\lambda_{1} / 2.8, I_{2}=I_{1} / 5-I_{1} / 3, \varphi_{2}=1.6 \pi-1.5 \pi$. 图 3 比较了气体靶中心处总强度为 $3.0 \times 10^{14} \mathrm{~W} / \mathrm{cm}^{2}$ 时, 基频场波长分别为 800 和 $2000 \mathrm{~nm}$ 的单色场和优化 场在长为 $1 \mathrm{~mm}$ 、压强为 3 Torr、位置距离激光焦点 $1.5 \mathrm{~mm}$ 处的 $\mathrm{Ne}$ 气喷嘴中产生的宏观谐波谱. 双色场 中, 两束激光脉冲的脉宽相等 $(\mathrm{FWHM}=3 T, T$ 为基频 场光学周期). 可以看出, 在保持激光能量不变的前提 下, 两种波长双色场中的谐波场都提高了 1 个数量级 以上. 需要指出的是, 这种双色场并不能改善谐波场 强度随激光波长变化的标度律 ${ }^{[86]}$. 最新的模拟结果 表明, 这种波形优化的方法也可以用于中空波导管 产生高强度的 $\mathrm{HHG}^{[87]}$.

根据谐波截止位置的理论公式 $E_{\text {cutoff }}=I_{p}+$ $3.17 U_{p}\left(U_{p} \sim I \lambda^{2}\right)$ 可知, 不同参数 $(I, \lambda)$ 的激光脉冲 都会产生相同截止位置的谐波. 应如何选择这些参 数来产生最强的谐波场? 在满足相位匹配的条件下 (电离 $<2 \%$ ), 短波长、高强度的激光产生的谐波场强 度更强. Jin 等人 ${ }^{[88]}$ 优化得到了截止位置在 $0.2-$ $1 \mathrm{keV}$ 能量范围内最优的双色场 $(\lambda+\lambda / 3)$ 波形. 利 用优化的双色场能够产生最强的谐波场: 与最优的 单色场相比, 谐波场强度提高 1 个数量级以上.
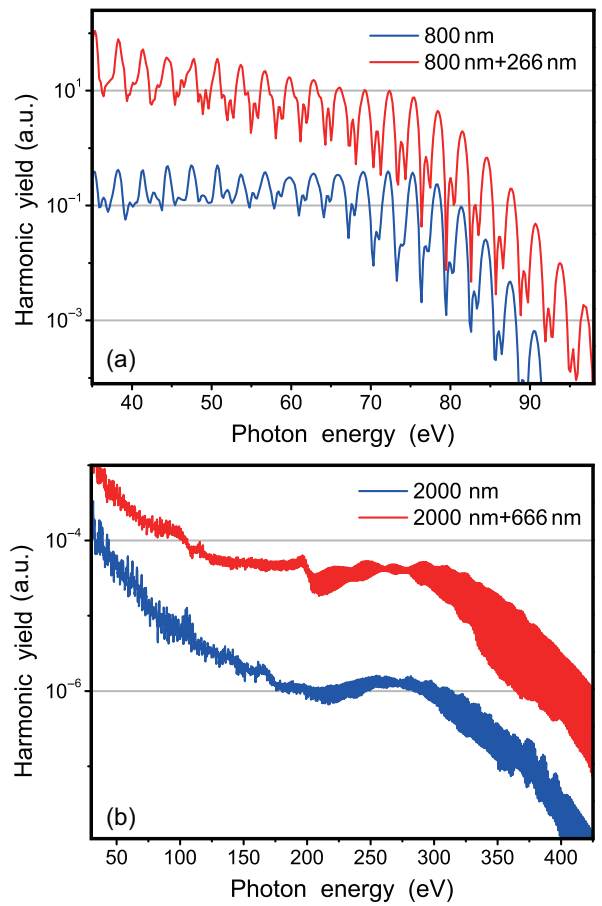

图 3 (网络版彩图) 气体靶中心处激光场总强度为 $3.0 \times 10^{14} \mathrm{~W} / \mathrm{cm}^{2}$ 时, 基频场波长为 800 (a) 和 $2000 \mathrm{~nm}$ (b) 的单 色场和优化双色场产生的 $\mathrm{Ne}$ 气宏观高次谐波谱的比较. 气体 靶长为 $1 \mathrm{~mm}$, 压强为 3 Torr, 放置在激光焦点 $1.5 \mathrm{~mm}$ 之后. 双 色场的参数为 ${ }^{[86]}: I_{2}=I_{1} / 5, \lambda_{2}=\lambda_{1} / 3, \varphi_{2}=1.6 \pi, \tau_{1}=\tau_{2}=3 T(T$ 为基频场光学周期). 经过优化后, 谐波场强度提高了 1 个数量 级以上

Figure 3 (Color online) The comparison of macroscopic HHG spectra generated from a 1-mm Ne gas get by single- and optimized two-color laser field, for fundamental wavelength of 800 (a) and $2000 \mathrm{~nm}$ (b), respectively. The total laser intensity is $3.0 \times 10^{14} \mathrm{~W} / \mathrm{cm}^{2}$. For the waveform of two-color field, $I_{2}=I_{1} / 5, \lambda_{2}=\lambda_{1} / 3, \varphi_{2}=1.6 \pi$.

通过优化激光场的波形, 还可以产生超强的 连续谐波谱, 进而获得超强、超短单个阿秒脉 冲 [71,85,89-91]. 例如, 如图 4 所示, 通过优化波长和 相位, 三束多周期 $(16 \mathrm{fs})$ 的激光场组合后会合成一 个亚周期脉冲 ${ }^{[89]}$. 利用这种激光场产生的阿秒脉冲 的强度会提高 $2-3$ 个数量级.

\subsection{2 扩展截止位置}

在基频场中加入一束长波红外脉冲可以扩展谐 波的截止位置 ${ }^{[82]}$. 国内工作者在此方面开展了很 多研究工作, 例如参见文献 [92-102]. 如果采用最 优的波形, 在不降低谐波场强度的前提下 (图 1(c)), 谐波的截止位置最大能提高多少倍? Chipperfield 等 
人 [103] 为此推导得到了一个理想波形: $E(t) \propto\left(\frac{3 t}{T}-\right.$ $1), T$ 为周期. 在该种波形的电场中, 电子获得的最大 返回动能是在相同能量单色场中的 3.11 倍. 他们提 出了可以利用具有倍频关系的五色优化场来实现该 种波形. 图 5 解释了返回动能增加的原因. 可以看出, 为了获得更高能量, 具有最大返回动能电子在单色 场和优化场中的运动时间由 $0.65 T$ 增大到 $0.92 T(T$ 为单色场的周期), 同时, 电离速率也得到了增强来弥 补波包扩散. 从能量的角度来看, 激光场波形改变后, 更多的电场能量用于电子的反向加速. 最近的实验

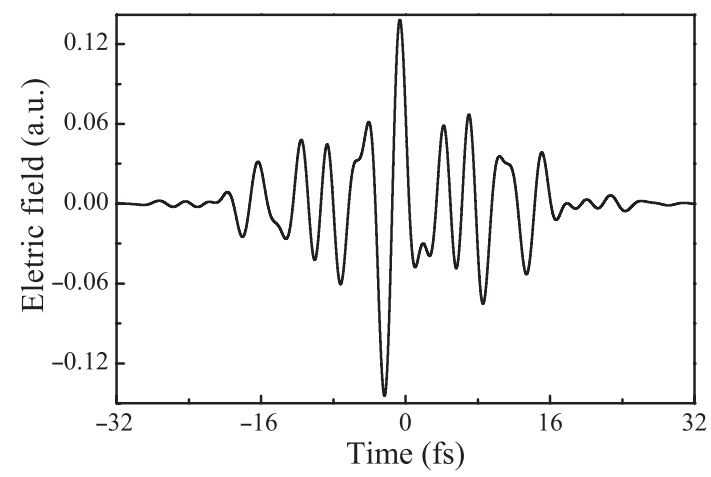

图 4 三束 $16 \mathrm{fs}$ 的非倍频光 $(1600+1168+792 \mathrm{~nm})$ 的激光场经 过组合后可合成一个亚周期的脉冲 [89]

Figure 4 Electric field resulting from the combination of three fields corresponding to 1600, 1168 and $792 \mathrm{~nm}$ [89]. Each field has a Gaussian envelope with duration of $16 \mathrm{fs}$ FWHM.

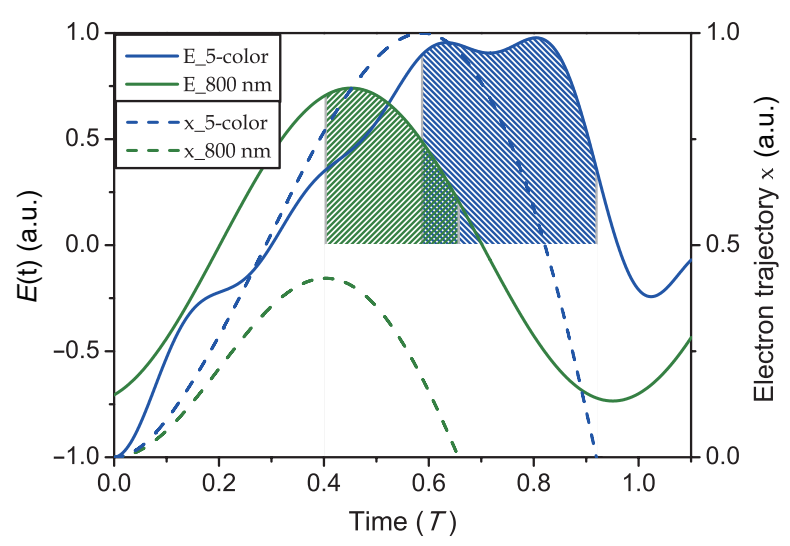

图 5 (网络版彩图) 单色场和优化五色场以及两种电场中最 大返回动能电子路径的比较 [105]

Figure 5 (Color online) Comparison of the electric fields of a pure sinusoidal and optimized 5-color field, and electron trajectories with maximum recollision energy are generated in two fields [105].
研究已经证明了利用三色场 $(1030+515+1545) \mathrm{nm}$ 合 成上升式激光场在提高谐波转换效率和截止位置方 面的可能性 ${ }^{[104]}$. 而我们的理论模拟则表明 (图 6), 采用更加可行的双色场 $\left(\lambda_{2} / \lambda_{1}=1.16, I_{2} / I_{1}=1, \varphi_{1}=\right.$ $\left.1.63 \pi, \varphi_{2}=0.13 \pi\right)$, 谐波的截止位置可提高 2 倍左右 (更多激光波长和气体压强下谐波谱的比较可参见文 献 [105]). 若在基频场中加入更多束低频场, 谐波截 止位置扩展得更显著 [106]. 在最近的一项工作中, Jin 等人 [107] 以广泛使用的 Ti:sapphire 激光 $(800 \mathrm{~nm})$ 作 为基频光, 对于不同的截止位置, 优化得到了第二束 激光 (中红外) 的参数 $\left(I_{2}, \lambda_{2}, \varphi_{2}\right)$.

\section{3 高次谐波动力学过程分析 - SST 时频 变换方法}

如果上述优化过程是为了按一定目的得到最优 的 HHG 谱, 那么, 为了理解强激光场与原子分子相 互作用产生高次谐波的机理就需要借助于动力学过 程分析, 为此可以对高次谐波进行时频分析 ${ }^{[108]}$, 并 结合经典的轨道分析探索高次谐波产生的动力学过 程. 常用的时频分析方法有 Morlet 变换 ${ }^{[109]}$, Gabor

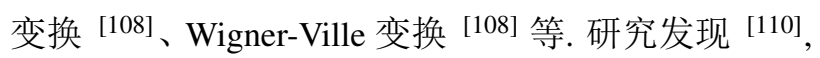
利用 Morlet 变换和 Gabor 变换获得的高次谐波时频 谱低能部分比较模糊, 而 Wigner-Ville 变换会得到一

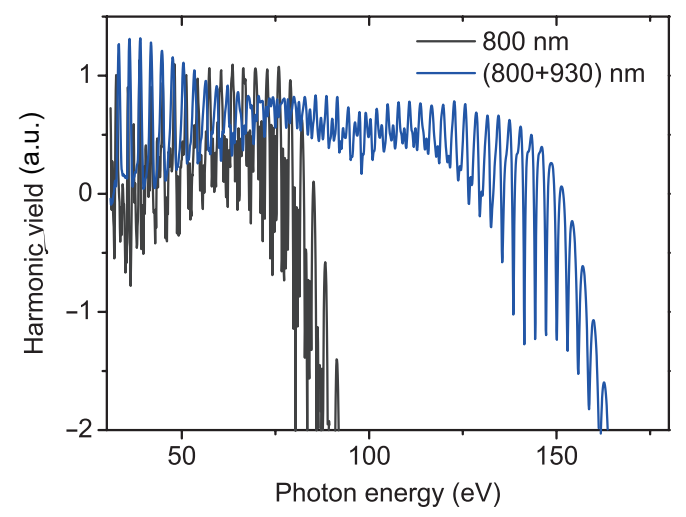

图 6 (网络版彩图) 单色场 $800 \mathrm{~nm}$ 和优化场 $(800+930) \mathrm{nm}$ 产 生 $\mathrm{Ne}$ 气宏观谐波谱的比较 ${ }^{[105]}$. 两种电场的能量相等, 谐波 场截止位置扩展了 2 倍

Figure 6 (Color online) The macroscopic harmonic spectra generated by a single $800 \mathrm{~nm}$ and optimized two-color $(800+930) \mathrm{nm}$ fields [105]. For the same laser fluence, the harmonic cutoff extension is about 2 times. 
些无法理解的结果, 这些都不利于高次谐波的动力 学过程分析. 最近发展起来的同步压缩时频变换技 术 $(\mathrm{SST})^{[35]}$ 可以用来分析高次谐波的动力学过程, 该方法能够克服其他变换方法获得的高次谐波时频 谱低能部分比较模糊的问题 [111]. SST 方法已经成 功应用于生物医学和化学领域 [112,113]. 下面将以铯 原子 (Cs) 为例, 通过同步压缩时频变换方法对高次 谐波的动力学过程进行分析.

SST 时频变换定义如下

$S(t, \xi)=\int V(t, \omega) \frac{1}{\alpha} h\left(\frac{\left|\xi-\omega_{f}(t, \omega)\right|}{\alpha}\right) \mathrm{d} \omega$,

其中 $\alpha>0, h(t)=\mathrm{e}^{-t^{2}} / \sqrt{\pi}$, 且 $V(t, \omega)$ 是修正的 Morlet 变换, 表示如下

$V(t, \omega)=\int A\left(t^{\prime}, \omega\right) g\left(t^{\prime}-t\right) \mathrm{e}^{-\mathrm{i} \omega\left(t^{\prime}-t\right)} \mathrm{d} t^{\prime}$,

$\omega_{f}(t, \omega)$ 是瞬时频率函数, 定义如下:

$\omega_{f}(t, \omega)= \begin{cases}\frac{-\mathrm{i} \partial V(t, \omega)}{V(t, \omega)}, & \text { for } \quad V(t, \omega) \neq 0, \\ \infty, & \text { for } \quad V(t, \omega)=0,\end{cases}$

其中, 修正的 Morlet 变换定义如下

$A_{\omega}\left(t^{\prime}, \omega\right)=\int d(t) \sqrt{\omega} W\left[\omega\left(t-t^{\prime}\right)\right] \mathrm{d} t$,

$W(x)$ 是窗函数

$W(x)=\frac{1}{\sqrt{\tau}} \mathrm{e}^{\mathrm{i} x} \mathrm{e}^{\frac{-x^{2}}{2 \tau^{2}}}$.

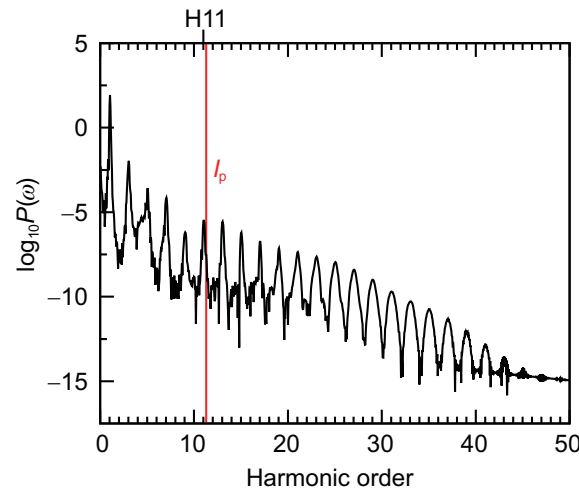

图 7 (网络版彩图) Cs 原子在 $3600 \mathrm{~nm}$ 的中红外激光场中产 生的高次谐波谱 ${ }^{[111]}$, 其中 $I_{\mathrm{p}}$ 表示 $\mathrm{Cs}$ 原子的基态电离能

Figure 7 (Color online) The HHG power spectrum of Cs driven by an intense 3600-nm (mid-infrared) laser pulse [111]. $I_{\mathrm{p}}$ is the atomic ionization potential of $\mathrm{Cs}$.
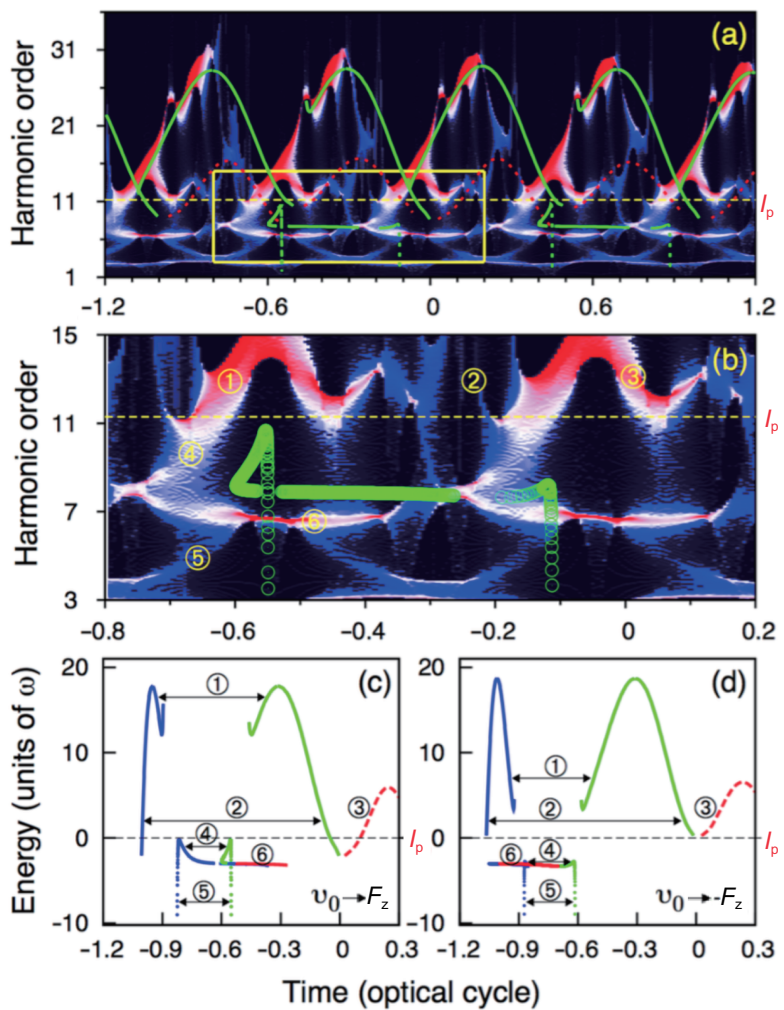

图 8 (网络版彩图) (a) Cs 原子在强激光场中产生高次谐波 的 SST 时频分析 ${ }^{[111]}$. 作为参考, 同时给出了经典模拟的结果, 如绿色线 (一次返回) 和红色线 (多次返回) 所示; (b) 近电离阈 值和电离阈值下 Cs 原子的高次谐波谱 SST 时频分析, 即图 (a) 黄色方框区域. (1), (2), (3)表示近电离阈值上的电子短轨道、长 轨道和多次返回轨道, (4), (5), (6)表示近电离阈值下的电子短 轨道、长轨道和共振位置附近的轨道; (c) 经典的电子返回能 量随电离时间和返回时间的变化关系, 其中电子初始速度 $v_{0}$ 顺着场力 $\boldsymbol{F}_{z}$ 方向; (d) 经典的电子返回能量随电离时间和返回 时间的变化关系, 其中电子初始速度 $v_{0}$ 逆着场力 $\boldsymbol{F}_{z}$ 方向. 其 他激光场参数同图 7

Figure 8 (Color online) (a) SST time-frequency spectra of the HHG from Cs atom in intense laser field [111]. For reference, the green curves and the red lines indicate the semiclassical trajectories of the first return and second return, respectively; (b) an enlarged view of the SST timefrequency profiles in the near- and below-threshold regions marked by the yellow window in (a). Here, (1), (2), (3) indicate the short trajectory, long trajectory, and multiple return trajectory in the near-threshold regions, and (4), (5), (6) indicate the short trajectory, long trajectory, and on-resonant trajectory in the below-threshold regions; (c) semiclassical return energy as a function of ionization time (blue lines) and return time (green lines and red dashed lines). Here the initial condition is that the electrons with an initial velocity $v_{0}$ are released along the electronic-field force $\boldsymbol{F}_{z}$. For clarity, we only show the semiclassical simulation for the electrons released during one optical cycle preceding the pulse peak; (d) same as $\mathrm{c}$, for the electrons with an initial velocity $v_{0}$ are released against the electronic-field force $\boldsymbol{F}_{z}$. The laser parameters used are the same as those in Figure 7. 
为了计算 $\mathrm{Cs}$ 原子的高次谐波谱, 构造了角动 量依赖的精确模型势 [111], 然后数值求解了含时薛 定谔方程 ${ }^{[114]}$, 获得了 Cs 原子在 $3600 \mathrm{~nm}$ 的中红 外激光场中产生的高次谐波谱, 如图 7 所示. 在计 算中, 激光场强度为 $I=1.4 \times 10^{12} \mathrm{~W} / \mathrm{cm}^{2}$, 持续时 间为 20 个周期. 对于 $\mathrm{Cs}$ 原子而言, 基态电离能为 0.143099 a.u. ${ }^{[111]}$, 相当于 11.3 个 $3600 \mathrm{~nm}$ 的光子能 量, 所以电离阈值为第 11 阶高次谐波 (H11).

图 8(a) 展示了 Cs 原子在强激光场中产生高次 谐波的 SST 时频分析结果, 由此可以获得高次谐波 的发射特性. 为了参考, 图中绿色线 (一次返回) 和红 色线 (多次返回) 表示出了经典模拟的结果. 可以看 出, 在电离阈值以上, 短轨道对产生高次谐波贡献较 大. 图 8(b) 展示了电离阈值附近及以下高次谐波的 时频谱, 其中, (1), (2), (3)表示近电离阈值上的电子短 轨道、长轨道和多次返回轨道, (4), (5), (6)表示近电离 阈值下的电子短轨道、长轨道和共振位置附近的轨 道. 可以看出, 多次返回轨道的贡献主要集中在第 9 阶和第 13 阶谐波之间, 共振增强的谐波发射位于第 7 阶谐波附近, 恰好和 Cs 原子的 6s-7p 的跃迁一致. 为了理解量子轨道在产生高次谐波过程中的角色, 进 行了经典的电子轨道模拟 (包含库仑势), 此时电子受 到的场力为 $\boldsymbol{F}_{z}=-E(t) z$ (原子单位), $E(t)$ 是激光场, $z$ 为激光场方向. 由于我们所使用的激光场参数在多 光子电离机制下 (Keldysh 参数 $\gamma=\sqrt{I_{\mathrm{p}} / 2 U_{\mathrm{p}}}=1.1$ ), 所 以选取了多光子电离的初始条件, 即电子拥有一个 初始速度顺着或逆着场力 $\boldsymbol{F}_{z}$ 的方向被释放. 当电子 沿着场力 $\boldsymbol{F}_{z}$ 方向被释放时, 电子可以获得额外的推 力而逃离势垒. 当电子逆着场力 $\boldsymbol{F}_{z}$ 方向被释放时, 电子被拉回势垒.

图 8(c) 和 (d) 展示了经典的电子返回能量随电 离时间和返回时间的变化关系, 其中 $v_{0}$ 为初始速度, $\boldsymbol{F}_{z}$ 为场力的方向. 当电子沿着场力 $\boldsymbol{F}_{z}$ 方向被释放时 (图 8(c)), 在近电离阈值以上, 电子的动力学过程表
现为短轨道(1), 长轨道(2), 以及多次返回轨道(3); 在 近电离阈值以下, 定义释放较早发射较晚的为长轨 道(4), 释放较晚发射较早的短轨道(5), 以及在共振位 置附近的轨道(6). 轨道(6)电子远离核后, 在 $0.1-0.3$ 个光学周期被立即拉回. 同时, 通过分析发现共振位 置附近的经典电子轨道对于沿着或逆着场力 $\boldsymbol{F}_{z}$ 方向 释放比较敏感, 逆着场力 $\boldsymbol{F}_{z}$ 方向释放的电子对近电 离阈附近多个谐波阶次发射有贡献, 而顺着场力 $\boldsymbol{F}_{z}$ 方向释放的电子仅对共振位置附近谐波的发射有贡 献. 总之, SST 时频分析的结果与经典模拟轨道有很 好的一致性, 两者结合分析, 可以为理解和探索电离 阈值附近及以下高次谐波发射的动力学过程提供了 强有力的工具.

\section{4 结论}

目前, 原子分子在强激光场中发射的高次谐波 是合成超短阿秒脉冲的主要方案之一, 高次谐波性质 的研究成为超快过程的热点研究领域, 已有很多重要 的研究成果, 限于篇幅, 不可能一一提及. 本文仅仅就 最近涉及的与高次谐波优化控制有关的问题以及常 用的飞秒激光脉冲整型技术在提高高次谐波转换效 率方面的应用作了一些介绍, 前期的进展在文献 [28] 中已有详细的论述. 随着激光技术的发展, 在实验室 已可以产生任意波形 (甚至亚周期) 的脉冲. 结合优 化理论, 这些激光脉冲在提高高次谐波的强度、扩展 谐波的截止位置方面具有非常明显的优越性, 使高 次谐波完全有可能成为被广泛利用的便捷型光源.

此外, 本文还介绍了最新发展的同步压缩时频 变换技术在分析高次谐波动力学过程中的应用. 该 时频变换技术在研究阈值附近 HHG 动力学过程中 显示出比其他小波变换更大的优越性, 相信在未来 会被广泛地用于强场物理研究中, 为揭示新的物理 机制提供重要的研究手段.

\section{参考文献}

1 Corkum P B. Plasma perspective on strong field multiphoton ionization. Phys Rev Lett, 1993, 71: 1994-1997

2 McPherson A, Gibson G, Jara H, et al. Studies of multiphoton production of vacuum-ultraviolet radiation in the rare gases. J Opt Soc Am B, 1987, 4: 595-601 
3 Ferray M, L'Huillier A, Li X F, et al. Multiple-harmonic conversion of 1064 nm radiation in rare gases. J Phys B, 1988, 21: L31

4 Salières P, L'Huillier A, Antoine P, et al. Study of the spatial and temporal coherence of high-order harmonics. Adv Atom Mol Opt Phys, 1999, 41: $83-142$

5 Zhao K, Zhang Q, Chini M, et al. Tailoring a 67 attosecond pulse through advantageous phase-mismatch. Opt Lett, 2012, 37: 3891-3893

6 Miao J W, Ishikawa T, Robinson I K, et al. Beyond crystallography: Diffractive imaging using coherent X-ray light sources. Science, 2015, 348: 530-535

7 Dinh K B, Le H V, Hannaford P, et al. Coherent diffractive imaging microscope with a high-order harmonic source. Appl Opt, 2015, 54: 5303-5308

8 Ravasio A, Gauthier D, Maia F R N C, et al. Single-shot diffractive imaging with a table-top femtosecond soft X-ray laser-harmonics source. Phys Rev Lett, 2009, 103: 028104

9 Plogmaker S, Terschlüsen J A, Krebs N, et al. HELIOS-A laboratory based on high-order harmonic generation of extreme ultraviolet photons for time-resolved spectroscopy. Rev Sci Instrum, 2015, 86: 123107

10 Dao L V, Dinh K B, Hannaford P. High order harmonic generation for study of atomic and molecular dynamics. Appl Phys Lett, 2013, 103: 141115

11 Grazioli C, Callegari C, Ciavardini A, et al. CITIUS: An infrared-extreme ultraviolet light source for fundamental and applied ultrafast science. Rev Sci Instrum, 2014, 85: 023104

12 La-O-Vorakiat C, Siemens M, Murnane M M, et al. Ultrafast demagnetization dynamics at themedges of magnetic elements observed using a tabletop high-harmonic soft X-ray source. Phys Rev Lett, 2009, 103: 257402

13 Vodungbo B, Barszczak S A, Gautier J, et al. Table-top resonant magnetic scattering with extreme ultraviolet light from high-order harmonic generation. EPL, 2011, 94: 54003

14 Weier C, Adam R, Rudolf D, et al. Femtosecond-laser-induced modifications in Co/Pt multilayers studied with tabletop resonant magnetic scattering. EPL, 2015, 109: 17001

15 Itatani J, Levesque J, Zeidler D, et al. Tomographic imaging of molecular orbitals. Nature, 2004, 432: 867-871

16 Shiner A D, Schmidt B E, Trallero H C, et al. Probing collective multi-electron dynamics in xenon with high-harmonic spectroscopy. Nat Phys, 2011, 7: 464-467

17 Wang G, Jin C, Le A T, et al. Conditions for extracting photoionization cross sections from laser-induced high-order-harmonic spectra. Phys Rev A, 2012, 86: 015401

18 Vampa G, Hammond T J, Thiré N, et al. All-optical reconstruction of crystal band structure. Phys Rev Lett, 2015, 115: 193603

19 Luu T T, Garg M, Kruchinin S Y, et al. Extreme ultraviolet high-harmonic spectroscopy of solids. Nature, 2015, 521: 498-502

20 Ye P, He X, Teng H, et al. Extraction of the in situ temporal information of few-cycle laser pulse from carrier-envelope phase-dependent high order harmonic spectrum. J Opt Soc Am B, 2014, 31: 1355-1359

21 Fana T, Grychtol P, Knut R, et al. Bright circularly polarized soft X-ray high harmonics for X-ray magnetic circular dichroism. Proc Nat Acad Sci, 2015, 17: 14206-14211

22 Fleischer A, Kfir O, Diskin T, et al. Spin angular momentum and tunable polarization in high-harmonic generation. Nat Photon, 2014, 8: 543-549

23 Kfir O, Grychtol P, Turgut E, et al. Generation of bright phase-matched circularly-polarized extreme ultraviolet high harmonics. Nat Photon, 2015, 9: $99-105$

24 Hickstein D D, Dollar F J, Grychtol P, et al. Noncollinear generation of angularly isolated circularly polarized high harmonics. Nat Photon, 2015, 9: $743-750$

25 Falcão-Filho E L, Lai C J, Hong K H, et al. Scaling of high-order harmonic efficiencies with visible wavelength drivers: A route to efficient extreme ultraviolet sources. Appl Phys Lett, 2010, 97: 061107

26 Rudawski P, Heyl C M, Brizuela F, et al. A high-flux high-order harmonic source. Rev Sci Instrum, 2013, 84: 073103

27 Hädrich S, Klenke A, Rothhardt J, et al. High photon flux table-top coherent extreme-ultraviolet source. Nat Photon, 2014, 8: 779-783

28 Winterfeldt C, Spielmann C, Gerber G. Optimal control of high-harmonic generation. Rev Mod Phys, 2008, 80: 117-140

29 Lewenstein M, Balcou P, Ivanov M Y, et al. Theory of high-harmonic generation by low-frequency laser fields. Phys Rev A, 1994, 49: 2117-2132

30 Jin C. Theory of Nonlinear Propagation of High Harmonics Generated in A Gaseous Medium. New York: Springer, 2013

31 Gaarde M B, Tate J L, Schafer K J. Macroscopic aspects of attosecond pulse generation. J Phys B, 2008, 41: 132001

32 Carroll D L. FORTRAN genetic algorithm driver, Version 1.7a. 2001

33 Werschnik J, Gross E K U. Quantum optimal control theory. J Phys B-At Mol Opt Phys. 2007, 40: R175-R211

34 Brif C, Chakrabarti R, Rabitz H. Control of quantum phenomena: Past, present and future. New J Phys, 2010, 12: 075008

35 Chen Y C, Cheng M Y, Wu H T. Non-parametric and adaptive modelling of dynamic periodicity and trend with heteroscedastic and dependent errors. J R Stat Soc B, 2014, 76: 651-682 
36 Weiner A M. Femtosecond pulse shaping using spatial light modulators. Rev Sci Instrum, 2000, 71: 1929-1960

37 Yao Y H, Lu C H, Xu S W, et al. Femtosecond pulse shaping technology and its applications (in Chinese). Acta Phys Sin, 2014, 63: 1842041 [姚 云华, 卢晨晖, 徐淑武, 等. 飞秒激光脉冲整形技术及其应用. 物理学报, 2014, 63: 1842041]

38 Bartels R, Backus S, Zeek E, et al. Shaped-pulse optimization of coherent emission of high-harmonic soft X-rays. Nature, 2000, 406: 164-166

39 Chu X, Chu S I. Optimization of high-order harmonic generation by genetic algorithm and wavelet time-frequency analysis of quantum dipole emission. Phys Rev A, 2001, 64: 021403(R)

40 Christov I P, Bartels R, Kapteyn H C, et al. Attosecond time-scale intra-atomic phase matching of high harmonic generation. Phys Rev Lett, 2001, 86: 5458-5461

41 Bartels R A, Murnane M M, Kapteyn H C, et al. Learning from learning algorithms: Application to attosecond dynamics of high-harmonic generation. Phys Rev A, 2004, 70: 043404

42 Pfeifer T, Walter D, Winterfeldt C, et al. Controlling the spectral shape of coherent soft X-rays. Appl Phys B, 2005, 80: 277-280

43 Spitzenpfeil R, Eyring S, Kern C, et al. Enhancing the brilliance of high-harmonic generation. Appl Phys A, 2009, 96: 69-81

44 Wei P, Miao J, Zeng Z, et al. Selective enhancement of a single harmonic emission in a driving laser field with subcyclewaveform control. Phys Rev Lett, 2013, 110: 233903

45 Wei P, Zeng Z, Jiang J, et al. Selective generation of an intense single harmonic from a long gas cell with loosely focusing optics based on a three-color laser field. Appl Phys Lett, 2014, 104: 151101

46 Wang X, Jin C, Lin C D. Coherent control of high-harmonic generation using waveform-synthesized chirped laser fields. Phys Rev A, 2014, 90: 023416

47 Reitze D H, Kazamias S, Weihe F, et al. Enhancement of high-order harmonic generation at tuned wavelengths through adaptive control. Opt Lett, 2004, 29: 86-88

48 Boyko O, Valentin C, Mercier B, et al. Systematic study of high-order harmonic optimal control by temporal pulse shaping of laser pulses. Phys Rev A, 2007, 76: 063811

49 Villoresi P, Bonora S, Pascolini M, et al. Optimization of high-order harmonic generation by adaptive control of a sub-10-fs pulse wave front. Opt Lett, 2004, 29: 207-209

50 Yoshitomi D, Nees J, Miyamoto N, et al. Phase-matched enhancements of high-harmonic soft X-rays by adaptive wave-front control with a genetic algorithm. Appl Phys B, 2004, 78: 275-280

51 Stefan E, Christian K, Michael Z, et al. Improving high-order harmonic yield using wavefront-controlled ultrashort laser pulses. Opt Express, 2012, 20: 5601-5606

52 Winterfeldt C, Pfeifer T, Walter D, et al. Adaptive temporal and spatial shaping of coherent soft X-rays. In: Proceedings of the International Society for Optical Engineering. Bellingham: SPIE, 2006

53 Salières P, L'Huillier A, Lewenstein M. Coherence control of high-order harmonics. Phys Rev Lett, 1995, 74: 3776-3779

54 Gaarde M B, Salin F, Constant E, et al. Spatiotemporal separation of high harmonic radiation into two quantum path components. Phys Rev A, 1999, 59: 1367-1373

55 Kim J H, Lee D G, Shin H J, et al. Wigner time-frequency distribution of high-order harmonics. Phys Rev A, 2001, 63: 063403

56 Chang Z, Rundquist A, Wang H, et al. Temporal phase control of soft-X-ray harmonic emission. Phys Rev A, 1998, 58: R30-R33

57 Lee D G, Kim J H, Hong K H, et al. Coherent control of high-order harmonics with chirped femtosecond laser pulses. Phys Rev Lett, 2001, 87: 243902

58 Li P C, Zhou X X, Wang G L, et al. Isolated sub-30-as pulse generation of an $\mathrm{He}^{+}$ion by an intense few-cycle chirped laser and its high-order harmonic pulses. Phys Rev A, 2009, 80: 053825

59 Niu Y, Xiang Y, Qi Y, et al. Single attosecond pulse generation from multicycle nonlinear chirped pulses. Phys Rev A, 2009, 80: 063818

60 Yedder B H A, Le B C, Atabek O, et al. Optimal control of attosecond pulse synthesis from high-order harmonic generation. Phys Rev A, 2004, 69: 041802(R)

61 Xiao J, Sun Z R, Wang Y F, et al. Optimization of high-order harmonic by genetic algorithm for the chirp and phase of few-cycle pulses. Chin Phys Lett, 2005, 22: 873-876

62 Xiao J, Sun Z R, Zhang X, et al. Optimization of single attosecond X-ray pulses by genetic algorithm control of the chirp and initial phase of 5 fs laser pulses. J Opt Soc Am B, 2006, 23: 771-775

63 Wang G, Jin C, Le A T, et al. Influence of gas pressure on high-order-harmonic generation of Ar and Ne. Phys Rev A, 2011, 84: 053404

64 Li X Y, Wang G L, Zhou X X. Effects of pressure and gas-jet thickness on the generation of attosecond pulse. Chin Phys B, 2014, 23: 013102

65 Roos L, Gaarde M B, L'Huillier A. Tailoring harmonic radiation to different applications using a genetic algorithm. J Phys B-At Mol Opt Phys, 2001, 34: 5041-5054 
66 Jan L, Stefan E, Robert S, et al. Maximizing the brilliance of high-order harmonics in a gas jet. New J Phys, 2009, 11: 023016

67 Wang L F, He X K, Teng H, et al. Tunable optimization of high-order harmonic generation driven by 5 fs laser pulses (in Chinese). Acta Phys Sin, 2014, 63: 224103 [汪礼锋, 贺新奎, 滕浩, 等. 5 fs 驱动激光脉冲的高次谐波选择性优化. 物理学报, 2014, 63: 224103]

68 Popmintchev T, Chen M C, Arpin P, et al. The attosecond nonlinear optics of bright coherent X-ray generation. Nat Photon, 2010, 4: 822-832

69 Yavuz D D. Toward synthesis of arbitrary optical waveforms. Science, 2011, 331: 1142-1143

70 Manzoni C, Mücke O D, Cirmi G, et al. Coherent pulse synthesis: Towards sub-cycle optical waveforms. Laser Photon Rev, 2015, 9: 129-171

71 Huang S W, Cirmi G, Moses J, et al. High-energy pulse synthesis with sub-cycle waveform control for strong-field physics. Nat Photon, 2011, 5: 475-479

72 Chan H S, Hsieh Z M, Liang W H, et al. Synthesis and measurement of ultrafast waveforms from five discrete optical harmonics. Science, 2011, 331: $1165-1168$

73 Mücke O D, Fang S, Cirmi G, et al. Toward waveform nonlinear optics using multimillijoule sub-cycle waveform synthesizers. IEEE J Sel Top Quantum Electron, 2015, 21: 8700712

74 Chen W J, Wang H Z, Lin R Y, et al. Attosecond pulse synthesis and arbitrary waveform generation with cascaded harmonics of an injection-seeded high-power $Q$-switched Nd:YAG laser. Laser Phys Lett, 2012, 9: 212-218

75 Watanabe S, Kondo K, Nabekawa Y, et al. Two-color phase control in tunneling ionization and harmonic generation by a strong laser field and its third harmonic. Phys Rev Lett, 1994, 73: 2692-2695

76 Kondo K, Kobayashi Y, Sagisaka A, et al. Tunneling ionization and harmonic generation in two-color fields. J Opt Soc Am B, 1996, 13: 424-429

77 Kim I J, Kim H T, Kim C M, et al. Efficient high-order harmonic generation in a two-color laser field. Appl Phys B, 2004, 78: 859-861

78 Mauritsson J, Johnsson P, Gustafsson E, et al. Attosecond pulse trains generated using two color laser fields. Phys Rev Lett, 2006, 97: 013001

79 Peng Y, Yang X, Zeng H, et al. Phase-matching control of high-order harmonic generation in a two-color laser field. Phys Rev A, 2007, 76: 063823

80 Takahashi E J, Lan P, Mücke O D, et al. Infrared two-color multicycle laser field synthesis for generating an intense attosecond pulse. Phys Rev Lett, 2010, 104: 233901

81 Bandulet H C, Comtois D, Bisson E, et al. Gating attosecond pulse train generation using multicolor laser fields. Phys Rev A, 2010, 81: 013803

82 Calegari F, Vozzi C, Negro M, et al. Efficient continuum generation exceeding 200 eV by intense ultrashort two-color driver. Opt Lett, 2009, 34: 3125-3127

83 Siegel T, Torres R, Hoffmann D J, et al. High harmonic emission from a superposition of multiple unrelated frequency fields. Opt Express, 2010, 18: 6853-6862

84 Negro M, Vozzi C, Kovacs K, et al. Gating of high-order harmonics generated by incommensurate two-color mid-IR laser pulses. Laser Phys Lett, 2011, 8: 875-879

85 Jin C, Wang G L, Wei H, et al. Waveforms for optimal sub-keV high-order harmonics with synthesized two- or three-colour laser fields. Nat Commun, 2014, 5: 4003

86 Wang G L, Dong X M, Zhao S F, et al. The high-harmonic conversion efficiencies in optimized two-color laser pulses (in Chinese). Chin Sci Bull, 2016, 61: 1808-1815 [王国利, 董小敏, 赵松峰, 等. 优化双色激光场产生高次谐波转化效率的理论研究. 科学通报, 2016, 61: 1808-1815]

87 Jin C, Stein G J, Hong K H, et al. Generation of bright, spatially coherent soft X-ray high harmonics in a hollow waveguide using two-color synthesized laser pulses. Phys Rev Lett, 2015, 115: 043901

88 Jin C, Wang G L, Le A T, et al. Route to optimal generation of soft X-ray high harmonics with synthesized two-color laser pulses. Sci Rep, 2014, 4: 7067

89 Li F, Wang G L, Zhao S F, et al. Synthesis of multi-color long laser pulses for strong attosecond pulse generation. Chin Phys Lett, 2015, 32: 014207

90 Balogh E, Bódi B, Tosa V, et al. Genetic optimization of attosecond-pulse generation in light-field synthesizers. Phys Rev A, 2014 , 90 : 023855

91 Chou Y, Li P C, Ho T S, et al. Optimal control of high-order harmonics for the generation of an isolated ultrashort attosecond pulse with two-color midinfrared laser fields. Phys Rev A, 2015, 91: 063408

92 Zeng Z, Cheng Y, Song X, et al. Generation of an extreme ultraviolet supercontinuum in a two-color laser field. Phys Rev Lett, 2007, 98 : 203901

93 Zhai Z, Yu R F, Liu X S, et al. Enhancement of high-order harmonic emission and intense sub-50-as pulse generation. Phys Rev A, 2008, 78: 041402(R)

$94 \mathrm{Du} \mathrm{H}$, Wang H, Hu B. Isolated short attosecond pulse generated using a two-color laser and a high-order pulse. Phys Rev A, 2010, 81: 063813

95 Luo J, Hong W, Zhang Q, et al. Dramatic cutoff extension and broadband supercontinuum generation in multi-cycle two color pulses. Opt Express, 2012, 20: 9801-9809 
96 Wang B, Li X, Fu P. Extension of the high-order-harmonic-generation cutoff frequency by two-color excitation. Phys Rev A, 2000, 62: 063816

97 Shao T, Zhao G, Wen B, et al. Theoretical exploration of laser-parameter effects on the generation of an isolated attosecond pulse from two-color high-order harmonic generation. Phys Rev A, 2010, 82: 063838

98 Xiang Y, Niu Y P, Gong S Q. Control of the high-order harmonics cutoff through the combination of a chirped laser and static electric field. Phys Rev A, 79: 053419

99 Guo Y H, He H X, Liu J Y, et al. Enhancement and extension of high-order harmonic emission and an isolated sub-100 as pulse generation from two-color laser fields. J Theor Comput Chem, 2010, 9: 735-744

100 Feng L Q, Chu T S. High-order harmonics extension and isolated attosecond pulse generation in three-color field: Controlling factors. Phys Lett A, 2011, 375: 3641-3648

101 Zhang G T, Bai T T, Zhang M G. Extension of harmonic cutoff and generation of isolated sub-30 as pulse in a two-color chirped laser field. Commun Theor Phys, 2012, 58: 557-564

102 Zhao D, Li F L. Extension of high-order harmonic cutoff frequency by synthesizing the waveform of a laser field via the optimization of classical electron trajectory in the laser field. Chin Phys B, 2013, 22: 064215

103 Chipperfield L E, Robinson J S, Tisch J W G, et al. Ideal waveform to generate the maximum possible electron recollision energy for any given oscillation period. Phys Rev Lett, 2009, 102: 063003

104 Haessler S, Balčiunas T, Fan G, et al. Optimization of quantum trajectories driven by strong-field waveforms. Phys Rev X, 2014, 4: 021028

105 Wang G L, Zhou L H, Zhao S F, et al. Synthesis of two-color laser pulses for the harmonic cutoff extension. Commun Theor Phys, 2016, 65: $601-605$

106 Solanpää J, Budagosky J A, Shvetsov-Shilovski N I, et al. Optimal control of high-harmonic generation by intense few-cycle pulses. Phys Rev A, 2014, 90: 053402

107 Jin C, Hong K H, Lin C D. Optimal generation of high harmonics in the water-window region by synthesizing 800-nm and mid-infrared laser pulses. Opt Lett, 2015, 40: 3754-3757

108 Flandrin P. Time-Frequency/Time-Scale Analysis. Pittsburgh: Academic Press, 1999

109 Tong X M, Chu S I. Probing the spectral and temporal structures of high-order harmonic generation in intense laser pulses. Phys Rev A, 2000, 61: $021802 \mathrm{R}$

110 Sheu Y L, Hsu L Y, Wu H T, et al. A new time-frequency method to reveal quantum dynamics of hydrogen in intense laser pulses: Synchrosqueezing Transform. AIP Adv, 2014, 4: 117138

111 Li P C, Sheu Y L, Laughlin C, et al. Dynamical origin of near- and below-threshold harmonic generation of Cs in an intense mid-infrared laser field. Nat Commun, 2015, 6: 7178

112 Suprunenko Y F, Clemson P T, Stefanovska A. Chronotaxic systems: A new class of self-sustained nonautonomous oscillators. Phys Rev Lett, 2013, 111: 024101

113 Wu H T, Chan Y H, Lin Y T, et al. Using synchrosqueezing transform to discover breathing dynamics from ECG signals. Appl Comput Harmon Anal, 2014, 36: 354-359

114 Tong X M, Chu S I. Theoretical study of multiple high-order harmonic generation by intense ultrashort pulsed laser fields: A new generalized pseudospectral time-dependent method. Chem Phys, 1997, 217: 119-130 


\title{
The optimal control and dynamical analysis of high-order harmonic generation from atom
}

\author{
WANG GuoLi ${ }^{1 *}$, LI PengCheng ${ }^{1 *}$, LI XiaoYong $^{2}$, ZHAO SongFeng $^{1} \&$ ZHOU XiaoXin $^{1 *}$ \\ ${ }^{1}$ College of Physics and Electronic Engineering, Northwest Normal University, Lanzhou 730070, China; \\ ${ }^{2}$ Experimental Center, Northwest University for Nationalities, Lanzhou 730030, China
}

\begin{abstract}
High-order harmonic generation (HHG) is a frequency up-conversion process in which an ultrafast femtosecond laser pulse at high intensity interacts with atom or molecule. HHG covers the wider wavelength spectral regions of extreme ultraviolet (XUV), soft X-ray and even hard X-ray. With the high photon flux, the HHG promises to be a attractive broadband tabletop light source, and a number of interesting applications of HHG have been demonstrated in many research fields. In this paper, we review some common methods and results on the optimal control of HHG to improve its low photon flux. HHG can now be controlled for different purposes, i.e., extending the cutoff energy, increasing the efficiency of the high-harmonic conversion process, and selecting single or range of harmonics, by combining the technologies of femtosecond temporal and spatial laser pulse shaping, waveform synthesizing and evolutionary algorithm, such as genetic algorithm and optimal control theory. Selective generation of a single harmonic can now be achieved both in hollow fiber, long gas cell and gas jet. We show that by using the newly developed waveform synthesis with two-color laser fields, the harmonic yield can be enhanced by more than one order without increasing the total laser energy, or the harmonic cutoff can be extended about two times without losing harmonic yield, even after considering the propagation effects. These progresses will exert a far-reaching impact on strong field physics. To optimize the HHG processes, a detailed understanding of the corresponding dynamics is essential. We also introduce a new time-frequency (TF) analysis technique, the synchrosqueezing transform (SST), which is used to reveal the quantum dynamics of HHG. Compared with the classical type of TF methods, such as the Gabor transform, the Morlet wavelet transform, the SST can be applied to explore the dynamical origin of near- and below-threshold harmonic emission.
\end{abstract}

high-order harmonic generation, optimal control, femtosecond pulse shaping, waveform synthesis, synchrosqueezing transform

PACS: $42.65 . \mathrm{Ky}, 42.65 . \mathrm{Re}, 32.80 . \mathrm{Qk}$

doi: $10.1360 /$ SSPMA2016-00251 